\title{
THE OCCURRENCE PROBLEM FOR MAPPING CLASS GROUPS
}

\author{
JOHN STILLWELL
}

\author{
(Communicated by Thomas J. Jech)
}

\begin{abstract}
The occurrence problem for the mapping class group $M(g, 0)$ of the closed orientable surface of genus $g$ is shown to be solvable for $g=1$ and unsolvable for $g>1$.
\end{abstract}

The occurrence problem for a finitely presented group $G$ is the problem of deciding, given $w, u_{1}, \ldots, u_{n} \in G$ (written as words in the generators of $G$ ), whether $w \in\left\langle u_{1}, \ldots, u_{n}\right\rangle$, the subgroup of $G$ generated by $u_{1}, \ldots, u_{n}$. Since the occurrence problem has the word problem as a special case (to decide whether $w=1$, ask whether $w \in\langle 1\rangle)$, it is also known as the generalized word problem. The latter term is due to Magnus, who solved the problem for one-relator groups in [6].

Mikhailova [9] showed that the occurrence problem is unsolvable for $F_{2} \times F_{2}$, the direct product of two copies of the free group $F_{2}$ of rank 2. Using this result, Makanina [8] showed that the occurrence problem is also unsolvable for the $n$ string braid groups $B_{n}$ when $n \geq 5$. Since braid groups are related to mapping class groups, one might expect a similar result for mapping class groups. The present paper confirms this, showing that the occurrence problem is unsolvable for $M(g, 0)$, the mapping class group of the closed orientable surface of genus $g$, when $g \geq 2$. As far as I know, this is the first appearance of unsolvability in surface topology (the conjugacy problem for mapping class groups was solved by Hemion [2]).

To show that the value of $g$ is best possible, we also show

THEOREM 1. The occurrence problem is solvable for $M(1,0)$, the mapping class group of the torus.

What makes $M(1,0)$ special is the fact that it has a certain quotient group for which the solution of the occurrence problem is already known, from [ 1 or 10]. Following a suggestion of the referee, I shall first show how solvability of the occurrence problem for a group $G$ follows from solvability of the occurrence problem for a quotient $G / N$ in fairly general circumstances.

In order to have a precise setting in which to employ algorithms, let us assume that the generators of $G / N$ are written as barred letters $\bar{x}$, where the letters $x$ are the generators in a fixed finite presentation of $G$. This implies, in particular, that we can go effectively from a word $\bar{w}=\bar{x}_{1} \bar{x}_{2} \cdots \bar{x}_{n}$ for an element of $G / N$ to a preimage $w=x_{1} x_{2} \cdots x_{n}$ in $G$, simply by erasing the bars. An instance of the occurrence problem for $G / N$ is a question whether $\bar{w} \in\left\langle\bar{u}_{1}, \ldots, \bar{u}_{n}\right\rangle$, where $\bar{w}, \bar{u}_{1}, \ldots, \bar{u}_{n}$ are

Received by the editors August 13, 1986.

1980 Mathematics Subject Classification (1985 Revision). Primary 20F10; Secondary 57M05.

Key words and phrases. Occurrence problem, mapping class group. 
words in the letters $\bar{x}$. A solution of the occurrence problem for $G / N$ is then an algorithm which processes any such words $\bar{w}, \bar{u}_{1}, \ldots, \bar{u}_{n}$ and correctly answers whether $\bar{w} \in\left\langle\bar{u}_{1}, \ldots, \bar{u}_{n}\right\rangle$. With this understanding of the problem and what its solvability means we assume

(1) The occurrence problem for $G / N$ is solvable.

A natural complement to this assumption is

(2) The occurrence problem for $G$ is solvable when restricted to instances $v \in$ $\left\langle u_{1}, \ldots, u_{n}\right\rangle$ with $v \in N$.

LEMMA. Under conditions (1) and (2), the occurrence problem for $G$ is solvable.

ProOF. Given an instance " $w \in\left\langle u_{1}, \ldots, u_{n}\right\rangle$ ?" of the occurrence problem for $G$, we first use the solution of the occurrence problem for $G / N$ to decide whether $\bar{w} \in\left\langle\bar{u}_{1}, \ldots, \bar{u}_{n}\right\rangle$. If this is not the case, then of course $w \notin\left\langle u_{1}, \ldots, u_{n}\right\rangle$.

If, on the other hand, $\bar{w} \in\left\langle\bar{u}_{1}, \ldots, \bar{u}_{n}\right\rangle$, then we can find a specific word $\bar{u}_{i_{1}} \cdots \bar{u}_{i_{k}}$ equal to $\bar{w}$ by enumerating all such words, and for each one testing whether $u_{i_{1}} \cdots \bar{u}_{i_{k}} \cdot \bar{w}^{-1} \in\langle 1\rangle$ until we get the answer yes, again using the solution of the occurrence problem for $G / N$. Having found $\bar{u}_{i_{1}} \cdots \bar{u}_{i_{k}}$, we compute the preimages $u_{i_{1}}, \ldots, u_{i_{k}}, w$ in $G$ of $\bar{u}_{i_{1}}, \ldots, \bar{u}_{i_{k}}, \bar{w}$, and consider the element

$$
u_{i_{1}} \cdots u_{i_{k}} w^{-1}=v \in N \text {. }
$$

Obviously, $v \in\left\langle u_{1}, \ldots, u_{n}\right\rangle \Rightarrow w \in\left\langle u_{1}, \ldots, u_{n}\right\rangle$ and conversely $v \notin\left\langle u_{1}, \ldots, u_{n}\right\rangle \Rightarrow$ $w=v^{-1} u_{i_{1}} \cdots u_{i_{k}} \notin\left\langle u_{1}, \ldots, u_{n}\right\rangle$. Hence $w \in\left\langle u_{1}, \ldots, u_{n}\right\rangle \Leftrightarrow v \in\left\langle u_{1}, \ldots, u_{n}\right\rangle$ and we can decide the latter question by assumption (2).

PROOF OF THEOREM 1 . It is a classical result that $M(1,0)=\operatorname{SL}(2, \mathbf{Z})$. A matrix $\left(\begin{array}{cc}m & n \\ p & q\end{array}\right) \in \mathrm{SL}(2, \mathbf{Z})$ faithfully represents the class of the torus mapping which sends the usual latitude curve $a$ and meridian curve $b$ to $m a+n b$ and $p a+$ $q b$ respectively. We write (homotopy classes of) curves additively here since the fundamental group of the torus is abelian. Conversely, all orientation-preserving torus mappings, whose classes comprise $M(1,0)$ by definition, are represented by matrices in $\mathrm{SL}(2, \mathbf{Z})$ (Tietze [12, p. 89]).

It is also known (see e.g. [7, p. 47]) that $\mathrm{SL}(2, \mathbf{Z})$ can be generated by the matrices

$$
S=\left(\begin{array}{cc}
1 & -1 \\
1 & 0
\end{array}\right) \quad \text { and } T=\left(\begin{array}{cc}
0 & 1 \\
-1 & 0
\end{array}\right)
$$

and that the following relations between $S$ and $T$ define $\operatorname{SL}(2, \mathbf{Z})$ abstractly:

$$
S^{3} T^{2}=T^{4}=1 .
$$

If $W$ is any element of $\operatorname{SL}(2, \mathbf{Z})$ and if $-W$ denotes the negative of $W$ (as a matrix), then the cosets $\bar{W}=\{ \pm W\}$ are the elements of the quotient group

$$
\operatorname{PSL}(2, \mathbf{Z})=\operatorname{SL}(2, \mathbf{Z}) /\{ \pm I\}
$$

where $I$ is the identity matrix. Since $S^{3}=T^{2}=-I$, we get $\bar{S}^{3}=\bar{T}^{2}=1$, and these are in fact the defining relations of $\operatorname{PSL}(2, \mathbf{Z})$, first found by Klein and Fricke [3, p. 454].

These relations show that $\operatorname{PSL}(2, \mathbf{Z})$ is the free product of $\mathbf{Z}_{3}=\left\langle\bar{S} ; \bar{S}^{3}=1\right\rangle$ and $\mathbf{Z}_{2}=\left\langle\bar{T} ; \bar{T}^{2}=1\right\rangle$, and Mikhailova [10] and Boydron [1] give algorithms for the occurrence problem in such free products. Thus Theorem 1 will follow from the 
Lemma if we can show that assumption (2) holds for the subgroup $N=\{ \pm I\}$ of $\mathrm{SL}(2, \mathbf{Z})$. This amounts to finding an algorithm which decides, given $u_{1}, \ldots, u_{n} \in$ $\mathrm{SL}(2, \mathbf{Z})$ (written as words in $S, T$ ), whether

$$
-I \in\left\langle u_{1}, \ldots, u_{n}\right\rangle \text {. }
$$

Finding this algorithm is the hard part of the proof, and it seems to depend heavily on the free product structure of $\operatorname{PSL}(2, \mathbf{Z})$.

In processing $u_{1}, \ldots, u_{n}$ we are guided by the behavior of their cosets $\bar{u}_{1}, \ldots, \bar{u}_{n}$ in $\operatorname{PSL}(2, \mathbf{Z})$. Since $\operatorname{PSL}(2, \mathbf{Z}) \simeq \mathbf{Z}_{3} * \mathbf{Z}_{2}$, the theorem of Kurosh [4] says that the subgroup $\left\langle\bar{u}_{1}, \ldots, \bar{u}_{n}\right\rangle$ is the free product of a free group $F$ and conjugates of the subgroups $\mathbf{Z}_{3}$ and $\mathbf{Z}_{2}$ generated by $\bar{S}$ and $\bar{T}$ (since the latter have no proper nontrivial subgroups). Lyndon [5] gives an algorithm for converting $\left\{\bar{u}_{1}, \ldots, \bar{u}_{n}\right\}$ to a basis $\left\{\overline{u_{1}^{*}}, \ldots, \overline{u_{m}^{*}}\right\}$ which reflects the free product structure of $\left\langle\bar{u}_{1}, \ldots, \bar{u}_{n}\right\rangle$. That is, $\overline{u_{m}^{*}}, \ldots, \overline{u_{m}^{*}}$ consists of free generators of $F$, together with generators of the other free factors of $\left\langle\bar{u}_{1}, \ldots, \bar{u}_{n}\right\rangle$. The latter generators are therefore conjugates of $\bar{S}$ or $\bar{T}$.

Lyndon's algorithm is a sequence of Nielsen transformations, hence the same sequence of transformations, applied to $\left\{u_{1}, \ldots, u_{m}\right\}$, will also give a generating set for $\left\langle u_{1}, \ldots, u_{n}\right\rangle$. The only point to bear in mind is that transformations which yield 1 as a generator in $\left\langle\bar{u}_{1}, \ldots, \bar{u}_{n}\right\rangle$ may yield $-I$ in $\left\langle u_{1}, \ldots, u_{n}\right\rangle$. The 1 will of course be omitted from the basis of $\left\langle\bar{u}_{1}, \ldots, \bar{u}_{n}\right\rangle$, whereas $-I$ must not be omitted from the generating set for $\left\langle u_{1}, \ldots, u_{n}\right\rangle$. However, if $-I$ arises in this way (as we can recognize by multiplying out the matrices which $S, T$ denote) we know immediately that $-I \in\left\langle u_{1}, \ldots, u_{n}\right\rangle$, and there is no need to continue with construction of $\left\{\overline{u_{1}^{*}}, \ldots, \overline{u_{m}^{*}}\right\}$. If it does not arise, then Lyndon's algorithm on $\left\{u_{1}, \ldots, u_{n}\right\}$ yields a generating set $\left\{u_{1}^{*}, \ldots, u_{m}^{*}\right\}$ of $\left\langle u_{1}, \ldots, u_{n}\right\rangle$ corresponding to the free product basis $\left\{\overline{u_{1}^{*}}, \ldots, \overline{u_{m}^{*}}\right\}$ of $\left\langle\bar{u}_{1}, \ldots, \bar{u}_{n}\right\rangle$.

Now if $-I \in\left\langle u_{1}, \ldots, u_{n}\right\rangle$ we have an equation

which implies

$$
-I=u_{i_{1}}^{*} u_{i_{2}}^{*} \cdots u_{i_{l}}^{*}
$$

Since the $\overline{u_{i}^{*}}$ form of a free product basis, the normal form theorem for elements of free products [7, p. 182] implies that the right-hand side of (i) contains a subword of the form either
(a) $\overline{u_{i}^{*}} \overline{u_{i}^{*}}-1$, or

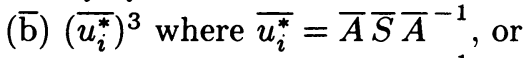

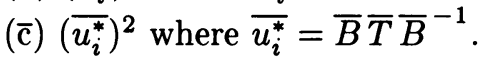

Consequently, the right-hand side of (i) contains a subword of the form either
(a) $u_{i}^{*} u_{i}^{*^{-1}}$, or
(b) $\left(u_{i}^{*}\right)^{3}$ where $u_{i}^{*}=A S A^{-1}$, or
(c) $\left(u_{i}^{*}\right)^{2}$ where $u_{i}^{*}=B T B^{-1}$.

By cancellation of any adjacent inverses (a), we see that (i) is only possible if the generating set $\left\{u_{i}^{*}, \ldots, u_{m}^{*}\right\}$ contains either an $A S A^{-1}$ or a $B T B^{-1}$. Conversely, if such an element is present, then an equation (i) holds, since

$$
\left(A S A^{-1}\right)^{3}=\left(B T B^{-1}\right)^{2}=-I .
$$




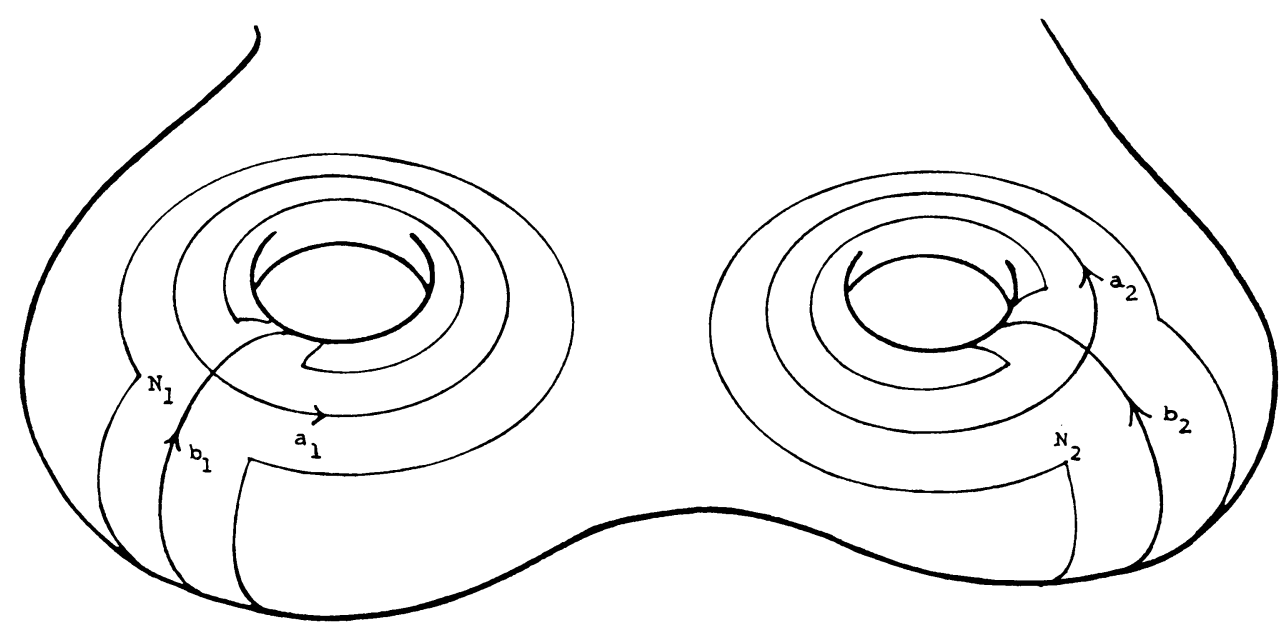

FIGURE 1

To sum up,

$$
\begin{aligned}
-I \in\left\langle u_{1}, \ldots, u_{n}\right\rangle & \Leftrightarrow \text { an element } A S A^{-1} \text { or } B T B^{-1} \in\left\{u_{i}^{*}, \ldots, u_{m}^{*}\right\} \\
& \Leftrightarrow \text { an element } \bar{A} \bar{S} \bar{A}^{-1} \text { or } \bar{B} \bar{T} \bar{B} \bar{u}^{-1} \in\left\{\overline{u_{i}^{*}}, \ldots, \overline{u_{m}^{*}}\right\} .
\end{aligned}
$$

Since we have an algorithm for producing $\left\{\overline{u_{i}^{*}}, \ldots, \overline{u_{m}^{*}}\right\}$, and we can decide whether any $\overline{u_{i}^{*}}$ is equal to an element $\bar{A} \bar{S} \bar{A}^{-1}$ or $\bar{B} \bar{T} \bar{B}^{-1}$ by the algorithm for the conjugacy problem in free products $[7$, p. 188], we have an algorithm for deciding whether $-I \in\left\langle u_{1}, \ldots, u_{n}\right\rangle$, as required.

The unsolvability result for $g>1$ which follows uses the topological interpretation of mapping classes, rather than a particular presentation of $M(g, 0)$. Thus it is necessary to mention that finite presentations of $M(g, 0)$ are known. The simplest published so far is in Wajnryb [13], and Wajnryb's generators in fact include the twist mappings used below.

THEOREM 2. The occurrence problem for $M(g, 0)$ is unsolvable when $g>1$.

Proof. Our method of proof is to show that $M(g, 0)$ contains $F_{2} \times F_{2}$ as a subgroup $H$ when $g>1$. Then there cannot be an algorithm for deciding occurrences of $w$ in the subgroup of $M(g, 0)$ generated by $u_{1}, \ldots, u_{n}$, otherwise we could apply it to any $w, u_{1}, \ldots, u_{n} \in H$ to solve the occurrence problem for $F_{2} \times F_{2}$, contrary to Mikhailova [9].

We can find two copies of $F_{2}$ in $M(g, 0)$ as follows. Take two distinct handles of the orientable surface $S_{g}$ of genus $g>1$ and consider the curves $a_{i}, b_{i}$ in each handle as shown in Figure 1. Let $G_{i}$ be the subgroup of $M(g, 0)$ generated by twist mappings along $a_{i}, b_{i}$. Thus each element of $G_{i}$ can be represented by a mapping which is the identity outside a union $N_{i}$ of annular neighborhoods of $a_{i}, b_{i}$. By choosing these neighborhoods to be sufficiently thin, we can ensure that $N_{1}$ is disjoint from $N_{2}$, as shown in Figure 1. Our representatives of $G_{1}$ then commute with our representatives of $G_{2}$. Consequently, each element of $G_{1}$ commutes with each element of $G_{2}$, and the subgroup of $M(g, 0)$ generated by $G_{1}$ and $G_{2}$ is just $G_{1} \times G_{2}$. 
To find an $F_{2} \times F_{2}$ in $M(g, 0)$ it therefore suffices to find an $F_{2}$ in each $G_{i} . G_{1}$ and $G_{2}$ are obviously isomorphic so we need look only at $G_{1}$. Now the twists $t_{a_{1}}$ and $t_{b_{1}}$ along $a_{1}$ and $b_{1}$ induce automorphisms of the homology group $H_{1}\left(S_{g}\right)$ of $S_{g}$ which can be faithfully represented by the matrices $\left(\begin{array}{ll}1 & 0 \\ 1 & 1\end{array}\right)$ and $\left(\begin{array}{ll}1 & 1 \\ 0 & 1\end{array}\right)$. Namely, $t_{a_{1}}$ sends $a_{1}$ to $a_{1}, b_{1}$ to $a_{1}+b_{1}$, and $t_{b_{1}}$ sends $a_{1}$ to $a_{1}+b_{1}, b_{1}$ to $b_{1}$, writing (homology classes of) curves additively; both are the identity on the remaining generators $a_{i}, b_{i}$ of $H_{1}\left(S_{g}\right)$. It follows that the subgroup of $G_{1}$ generated by $t_{a_{1}}^{2}$ and $t_{b_{1}}^{2}$ has a homomorphic image generated by

$$
\left(\begin{array}{ll}
1 & 0 \\
1 & 1
\end{array}\right)^{2}=\left(\begin{array}{ll}
1 & 0 \\
2 & 1
\end{array}\right) \text { and }\left(\begin{array}{ll}
1 & 1 \\
0 & 1
\end{array}\right)^{2}=\left(\begin{array}{ll}
1 & 2 \\
0 & 1
\end{array}\right) \text {. }
$$

However, the latter matrices are known to generate a free group, $F_{2}$ (this was essentially known to Klein and Fricke [3, p. 276], and an algebraic proof may be found in [7, p. 101]). Consequently $t_{a_{1}}^{2}$ and $t_{b_{1}}^{2}$ generate an $F_{2}$ in $G_{1}$, as required.

The referee has pointed out that an $F_{2} \times F_{2}$ in $M(g, 0), g \geq 2$, may also be found using known group-theoretic results. This arises from the identification of $M(g, 0)$ with the outer automorphism group of

$$
\pi_{1}\left(S_{g}\right)=\left\langle a_{1}, b_{1}, \ldots, a_{g}, b_{g} ; a_{1} b_{1} a_{1}^{-1} b_{1}^{-1} \cdots a_{g} b_{g} a_{g}^{-1} b_{g}^{-1}=1\right\rangle
$$

established in Nielsen [11].

First consider the full automorphism group $\operatorname{Aut}\left(\pi_{1}\left(S_{g}\right)\right)$ and, for each $i$, its subgroup $A_{i}$ which maps the subgroup $\left\langle a_{i}, b_{i}\right\rangle$ of $\pi_{1}\left(S_{g}\right)$ onto itself and fixes each $a_{j}$ and $b_{j}$ for $j \neq i$. Any two such $A_{i}$, say $A_{1}$ and $A_{2}$, obviously generate their own direct product in $\operatorname{Aut}\left(\pi_{1}\left(S_{g}\right)\right)$. That is, if $\alpha_{1} \in A_{1}$ and $\alpha_{2} \in A_{2}$ then $\alpha_{1} \alpha_{2}=\alpha_{2} \alpha_{1}$. It follows fairly easily that in the quotient $M(g, 0)=\operatorname{Aut}\left(\pi_{1}\left(S_{g}\right)\right) / \operatorname{Inn}$, where Inn is the group of inner automorphisms, the corresponding groups $G_{1}=A_{1} \operatorname{Inn} / \mathrm{Inn}$, $G_{2}=A_{2}$ Inn / Inn also generate their own product. This pair $G_{1}, G_{2}$ is the same as the pair defined topologically in Theorem 2 .

It now remains to show, using the algebraic definition, that each $G_{i}$ contains an $F_{2}$. One can either use theorems of Nielsen which show $A_{i} \operatorname{Inn} / \operatorname{Inn} \simeq \operatorname{SL}(2, \mathbf{Z})$ or else observe the action of $G_{i}$ on $H_{1}\left(S_{g}\right)$, which is the same as that of $A_{i}$, since inner automorphisms of $\pi_{1}\left(S_{g}\right)$ act trivially on its abelianization $H_{1}\left(S_{g}\right)$. It is clear algebraically, even more so than topologically, that the induced automorphisms of $H_{1}\left(S_{g}\right)$ include those represented by the matrices $\left(\begin{array}{ll}1 & 0 \\ 1 & 1\end{array}\right)$ and $\left(\begin{array}{ll}1 & 1 \\ 0 & 1\end{array}\right)$. Thus with either approach one obtains an $F_{2}$ from the matrices $\left(\begin{array}{ll}1 & 0 \\ 2 & 1\end{array}\right)$ and $\left(\begin{array}{ll}1 & 2 \\ 0 & 1\end{array}\right)$.

\section{REFERENCES}

1. Y. Boydron, Algorithmes dans les produits libres, C. R. Acad. Sci. Paris 282 (1976), A135A138.

2. G. Hemion, On the classification of homeomorphisms of 2-manifolds and the classification of 3-manifolds, Acta Math. 32 (1979), 123-155.

3. F. Klein and R. Fricke, Vorlesungen über die Theorie der elliptischen Modulfunktion, vol. I, Teubner, Stuttgart, 1890.

4. A. G. Kurosh, Die Untergruppen der freien Produkte von beliebigen Gruppen, Math. Ann. 109 (1934), 647-660.

5. R. Lyndon, Length functions in groups, Math. Scand. 12 (1963), 209-234.

6. W. Magnus, Das Identitätsproblem für Gruppen mit einer definierenden Relation, Math. Ann. 106 (1932), 295-307. 
7. W. Magnus, A. Karrass, and D. Solitar, Combinatorial group theory, Wiley-Interscience, New York, 1966.

8. T. A. Makanina, The occurrence problem in the braid group $B_{n+1}$ with $n+1 \geq 5$, Mat. Zametki 29 (1981), 31-33. (Russian)

9. K. A. Mikhailova, The occurrence problem for direct products of groups, Dokl. Akad. Nauk SSSR 119 (1958), 1103-1105. (Russian)

10. _ The occurrence problem for free products of groups, Mat. Sb. 75 (1968), 199-210.

11. J. Nielsen, Untersuchungen zur Topologie der geschlossenen zweiseitigen Flächen I, Acta Math. 50 (1927), 189-358.

12. $\mathrm{H}$ Tietze, Über die topologischen Invarianten mehrdimensionaler Mannigfaltigkeiten, Monatsh. Math. Phys. 19 (1908), 1-118.

13. B. Wajnryb, A simple presentation for the mapping class group of an orientable surface, Israel J. Math. 45 (1983), 157-174.

Department of Mathematics, Monash University, Clayton, Victoria 3168, Australia 